\title{
Variabilidade sazonal dos constituintes da própolis vermelha e bioatividade em Artermia salina
}

\author{
Lívio César Cunha Nunes, ${ }^{1}$ Alexandre Bezerra Galindo, ${ }^{3}$ Annalu da Silva Oliveira de Deus, ${ }^{1}$ \\ Daniel Arcanjo Rufino, ${ }^{1}$ Karina Perrelli Randau, ${ }^{2}$ Haroudo Satiro Xavier, ${ }^{2}$ Antônia Maria \\ das Graças Lopes Citó, ${ }^{1}$ Pedro José Rolim Neto ${ }^{* 3}$
}

\author{
${ }^{I}$ Núcleo de Tecnologia Farmacêutica, Universidade Federal do Piaui, 60400-000 Teresina-PI, Brasil, \\ ${ }^{2}$ Laboratório de Farmacognosia, Departamento de Ciências Farmacêuticas, Universidade Federal de \\ Pernambuco, 50740-521 Recife-PE, Brasil, \\ ${ }^{3}$ Laboratório de Tecnologia dos Medicamentos, Departamento de Ciências Farmacêuticas, Universidade Federal \\ de Pernambuco, 50740-521 Recife-PE, Brasil
}

\begin{abstract}
RESUMO: A própolis é uma substância resinosa coletada pelas abelhas de diversas partes das plantas. Sua composição depende da época, vegetação e local de coleta. Apresenta diversas atividades biológicas como antimicrobiana, antioxidante, antitumoral, dentre outras. Foi realizado estudo da variabilidade sazonal, nos meses de fevereiro, junho e outubro de 2006, dos constituintes voláteis da própolis vermelha de Pernambuco através da extração por headspace dinâmico e identificação por cromatografia gasosa acoplada com espectrometria de massas (CG-EM). Foram identificados 34 constituintes voláteis, sendo monoterpenos e monoterpenóides, sesquiterpenos e sesquiterpenóides, fenilpropanóides, aldeídos, cetonas e $\eta$-alcanos. Os constituintes majoritários foram o trans-anetol, $\alpha$-copaeno e o metil cis-isoeugenol. Também foi realizado o perfil fitoquímico por cromatografia em camada delgada (CCD), através da qual os constituintes fenólicos foram identificados como majoritários. Com o extrato bruto metanólico da própolis, realizou-se o ensaio de letalidade em Artemia salina, que demonstrou $\mathrm{DL}_{50}$ de $18,9 \mu \mathrm{g} / \mathrm{mL}$, sugerindo uma possível atividade antitumoral.
\end{abstract}

Unitermos: Própolis vermelha, constituintes voláteis, Artemia salina.

\begin{abstract}
Seasonal variability of red propolis constituents and brine shrimp bioassay". Propolis is a resinous hive product collected by honeybees from plant sources. The composition of the propolis depends on the season, the vegetation, and the area of collection. It presents a diverse biological activities such as antimicrobial, antioxidant and antitumoral. The seasonal variability of the red propolis constituents from Pernambuco State were accomplished in the months of February, June and October of 2006. The volatile red propolis constituients were captured by the dynamic headspace technique and analyzed by GC-MS. In the analysis of the samples, 34 constituents were identified as the mono and sesquiterpens, phenylpropanoids, aldehydes, cetons and $n$-alcanes, presenting as majority constituents the trans-anethol, $\alpha$-copaene and methyl cis-isoeugenol. The phytochemistry profile was performed by thin layer chromatography (TLC). Through this technique the majority constituents were identified as phenols. The Brine shrimp bioassay was used to evaluate of the red propolis methanolic extract, which demonstrated a $\mathrm{DL}_{50}$ of $18.9 \mu \mathrm{g} / \mathrm{mL}$ and an activity antitumoral was possibly suggested.
\end{abstract}

Keywords: Red propolis, volatile constituents, Artemia salina.

\section{INTRODUÇÃO}

A própolis é considerada uma das misturas mais heterogêneas encontradas em fontes naturais, sendo que mais de 300 constituintes já foram identificados e/ou caracterizados em diferentes amostras (Burdock, 1998; Chang et al., 2008; Lustosa et al., 2008; Sousa et al., 2008). As abelhas (Apis mellifera) usam esta substância para protegê-las contra insetos e microorganismos, empregando-a em finas camadas nas paredes internas das colméias para vedar buracos e rachaduras, reparar e fortalecer os favos de mel, proteger a entrada da colméia, no preparo de locais assépticos para a postura da abelha rainha e na mumificação de insetos invasores (Bankova et al., 2000).

A própolis é coletada por abelhas a partir de diversas partes das plantas como brotos, botões florais, casca e exsudatos resinosos (Park et al., 1997). As propriedades biológicas da própolis estão diretamente ligadas a sua composição química e este possivelmente é o 
maior problema para o uso da própolis na terapia, tendo em vista que a sua composição química varia com a flora da região, locais e épocas de coleta, com a técnica empregada para a produção (tipo de coletores) e com as características genéticas (gênero e espécie das abelhas). Este conjunto de fatores exerce, portanto, uma enorme importância nas suas propriedades físicas, químicas e biológicas (Pereira et al., 2002; Park et al., 1998; Bianchini \& Bedendo, 1998; Dutra et al., 2008; Nascimento et al., 2008; Simões et al., 2008).

No Brasil, são descritas propriedades biológicas e composição química distintas para diferentes amostras coletadas em diferentes partes do país. Essa variação é facilmente explicada pela grande biodiversidade brasileira (Pereira et al., 2002; Longhini et al., 2007). Com relação à variação sazonal, a diminuição em alguns componentes biologicamente ativos, como no caso dos fenólicos, é acompanhada pelo aumento de outros, por exemplo, ácidos diterpênicos. Deste modo, pode-se esperar que algumas atividades biológicas, relacionadas a estes compostos (antibacteriana, antifúngica), sejam similares em diferentes estações do ano (Bankova et al, 1998).

Park et al. (2002) classificaram a própolis brasileira em doze tipos de acordo com a região geográfica de origem, composição química e vegetação de onde foi extraída. Porém, a própolis vermelha, a qual é encontrada, sobretudo nas áreas litorâneas das regiões norte e nordeste, ainda é pouco estudada. O presente trabalho objetiva caracterizar os constituintes químicos, principalmente os voláteis, da própolis vermelha de Pernambuco e realizar o biomonitoramento através da avaliação de sua citotoxicidade sobre Artemia salina Leach.

\section{MATERIAL E MÉTODOS}

As amostras foram coletadas numa área de restinga, cidade de Goiana-PE (distrito de Atapuz), nos meses de fevereiro, junho e outubro de 2006, acondicionadas em recipiente plástico opaco, hermeticamente fechado e conservadas sob refrigeração.

\section{Identificação dos constituintes voláteis}

Cada uma das amostras de própolis $(7,5 \mathrm{~g})$ foram submetidos à técnica de headspace dinâmico por duas horas, utilizando-se como armadilha para a captura dos voláteis o Porapak-Q® (divinilbenzeno/etilvinilbenzeno). Os constituintes voláteis foram dessorvidos do polímero pela adição de diclorometano, que em seguida foi eliminado por um jato de nitrogênio. Posteriormente, a análise dos constituintes foi realizada num cromatógrafo Shimadzu GC-17A acoplado a um espectômetro de massas GCMSQP5050A equipado com uma coluna capilar de sílica fundida DB-5 (95\% polidimetilsiloxano e 5\% difenil, 30 $\mathrm{m}$ de comprimento, 0,25 $\mathrm{mm}$ de diâmetro externo, 0,25 $\mu \mathrm{m}$ de diâmetro interno). A programação utilizada para injeção e corrida das amostras foi a seguinte: injetor: 220
${ }^{\circ} \mathrm{C}$, detetor: $240{ }^{\circ} \mathrm{C}$, coluna: $60{ }^{\circ} \mathrm{C}$ a $240{ }^{\circ} \mathrm{C}, 3{ }^{\circ} \mathrm{C} \cdot \mathrm{min}^{-1} . \mathrm{O}$ gás de arraste foi hélio com vazão de $1 \mathrm{~mL}$. $\mathrm{min}^{-1}$.

A identificação dos constituintes foi realizada por inspeção visual dos seus espectros de massas comparandoos com espectros da literatura (Adams, 1995), observando principalmente os parâmetros pico do íon molecular e pico base, além de comparação com espectros de uma biblioteca computacional (Wiley 299) através da comparação dos índices de Kovats calculados em co-injeção de uma série homóloga de $n$-alcanos, com os índices de Kovats encontrados na literatura (Adams, 1995).

\section{Perfil fitoquímico da própolis através da CCD}

Própolis ( $1 \mathrm{~g})$, triturado, de cada um dos três meses de coleta foi submetido à extração metanólica (20 mL). Alíquotas de $10 \mu \mathrm{L}$ foram analisadas por cromatografia em camada delgada (placas de gel de sílica Merck - Alemanha, art.105554) para análise da presença de grupos de metabólitos, empregando-se diversas fases móveis e reveladores adequados (Harborne, 1984; Wagner \& Bladt, 1996; Sena Filho et al., 2006).

\section{Bioensaio de letalidade sobre Artemia salina}

O ensaio de toxicidade sobre a Artemia salina Leach foi realizado de acordo com a metodologia proposta por Meyer et al. (1982), utilizando $1 \mathrm{~g}$ da própolis, que foi submetido à extração em metanol por 24 horas sob agitação (EB-MeOH). A partir de $20 \mathrm{mg}$ do extrato seco, foram preparadas soluções de 1, 3, 10, 30, 100, 300 e $1000 \mu \mathrm{g} /$ $\mathrm{mL}$, em triplicata, que foram ressuspensas em clorofórmio $\left(\mathrm{CHCl}_{3}\right)$ e $0,1 \mathrm{~mL}$ de dimetilsulfóxido (DMSO). Para o teste de letalidade, 10 espécimes do microcrustáceo, com 48 horas de eclosão em água do mar e água destilada (1:1), foram colocados em cada um dos tubos e em três tubos controle (clorofórmio + DMSO). Após 24 horas, contouse o número de mortos e os resultados obtidos foram tabulados e submetidos à análise de Probitos no software estatístico SPSS ${ }^{\circledR}$ for Windows 10.0, obtendo-se o valor da $\mathrm{DL}_{50}$, com intervalo de confiança a $95 \%$.

\section{RESULTADOS E DISCUSSÕES}

\section{Constituintes voláteis}

Através da técnica de headspace dinâmico e da análise por CG-EM, foram identificados 34 constituintes voláteis, considerando as três amostras da própolis vermelha. A Tabela 1 apresenta os constituintes voláteis identificados de acordo com a classe química. Pelos resultados, evidencia-se uma composição química em voláteis bastante variada, destacando-se como constituintes majoritários trans-anetol, $\alpha$-copaeno e metil cis-isoeugenol. O trans-anetol, bem como o elemicin, foram apresentados em estudo recente por Marcucci et al.

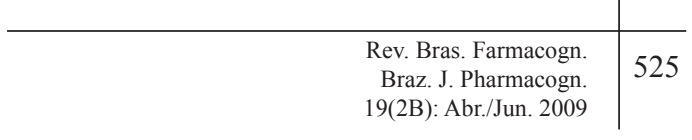


(2006) como componentes majoritários de uma amostra de própolis vermelha de Maceió-Alagoas, o que nos leva a correlacionar as origens comuns para esse tipo de própolis. O elemicin foi citado como componente da própolis pela primeira vez no estudo de Marcucci et al. (2006). Nesse estudo, nas três amostras de própolis, o fenilpropanoide elemicin foi também identificado. Petri et al. (1988) classificaram a própolis de clima temperado em dois tipos, conforme o constituinte volátil majoritário o ß-eudesmol ou o benzoato de benzila. Vários estudos evidenciaram que a própolis destas regiões origina-se principalmente de botões florais de plantas da espécie Populus (Bankova, 2000). Nos países de clima tropical, a composição química da própolis é de difícil caracterização, dada a grande diversidade da flora e por sua composição química diferir bastante da própolis produzida em regiões de clima temperado.

A variação na composição química em voláteis, entre as três amostras coletadas, em diferentes épocas do ano, foi pequena, havendo dezessete constituintes comuns dentre os compostos identificados. Contudo, apenas na amostra coletada em outubro foi evidenciado o sesquiterpenóide $\delta$-cardinol e os sesquiterpenos $\beta$-gurjuneno, isocariofileno e $\delta$-cadineno. Os $n$-alcanos foram identificados na amostra do mês de junho, assim como o monoterpenóide 1,8 cineol e o sesquiterpeno $\alpha$-selineno.

No entanto, vale ressaltar que o constituinte majoritário foi o mesmo nas três amostras, o trans-anetol, e que o percentual deste composto foi relativamente alto, particularmente na amostra coletada em outubro $52,58 \%$. Também se destaca nas três amostras o alto teor de fenilpropanóides, sendo aproximadamente $66 \%$ para a amostra coletada em outubro. Os constituintes dos óleos essenciais pertencem a dois grupos de metabólitos originados de rotas biossintéticas diferentes: o grupo dos terpenos, cuja rota biossintética deriva de unidades de isopreno; e o grupo dos compostos aromáticos, derivados dos fenilpropanóides e que tem como precursor o ácido chiquímico (Dewick, 2003). A amostra coletada no mês de outubro corresponde à estação após o período das chuvas no Nordeste, época em que a maioria das espécies vegetais nativas floresce e por isso a produção de própolis é maior nesta época do ano. Na estação das chuvas, as abelhas visitam arbustos e subarbustos, enquanto que no período seco as espécies lenhosas são as principais fontes de néctar, pólen e resina para as abelhas, mostrando assim uma mudança no pasto apícola e, conseqüentemente, uma variação na composição química da própolis. Ainda nas três amostras estudadas observase grande percentual de sesquiterpenos. Esses compostos, juntamente com fenilpropanóides, são responsáveis por diversas atividades farmacológicas, com destaque para o constituinte majoritário trans-anetol, o qual apresenta atividades comprovadas, tais como: analgésica, anestésica, antigenotóxica, antioxidante, dentre outras (Nirmala et al, 2005). Este se mostrou ainda como sendo o principal constituinte do óleo essencial de anis (Pimpinella anisum), dotado de atividade inseticida e antifúngica (Costa, 2002; Moersdorf, 1966; Shukla \& Tripathi, 1987). Essas e outras atividades atribuídas aos demais constituintes indicam um bom potencial farmacológico para a própolis em estudo.

\section{Análise fitoquímica}

A cromatografia em camada delgada mostrou um mesmo perfil fitoquímico para a própolis entre os meses de coleta. Os metabólitos secundários majoritários da própolis foram os derivados fenólicos (flavonóides, antraquinonas) e terpenos (monoterpenos, sesquiterpenos, triterpenos e esteróides), além da presença de açúcares. Não foi detectada a presença de alcalóides, iridóides, saponinas, cumarinas, derivados cinâmicos, fenilpropanoglicosídeos, proantocianidinas condensadas e leucoantocianidinas. Flavonóides como quercetina, kaempferol, luteolina e apigenina, também não foram detectados, mesmo sendo reportados na literatura em outras própolis (Pietra et al., 2002; Salatino et al., 2005). Porém, há uma grande controvérsia em relação ao teor de flavonóides nas amostras brasileiras (Pereira et al., 2002). Bankova et al. (1995) concluíram que as própolis brasileiras têm uma baixa concentração de flavonóides e ésteres de ácidos fenólicos, possuindo altas concentrações de ácido dihidroxicinâmico, acetofenonas preniladas e alguns terpenóides específicos. Ainda Bankova et al. (2000) citam que em alguns casos os flavonóides são importantes componentes presentes na própolis brasileira. Aga et al. (1994) determinam os compostos prenilados como os maiores constituintes da própolis brasileira e relatam que a atividade antibacteriana destes compostos pode ser aumentada com o aumento do número de resíduos prenil na molécula, como citado por Marcucci et al. (2001).

\section{Bioensaio com Artemia salina Leach}

No ensaio de toxicidade sobre Artemia salina, o extrato bruto metanólico da própolis apresentou $\mathrm{DL}_{50}$ igual a $18,9 \mu \mathrm{g} / \mathrm{mL}$, com intervalo de confiança a $95 \%$ entre $14,6-24,8 \mu \mathrm{g} / \mathrm{mL}$. Este resultado sugere uma atividade antitumoral, devido a $\mathrm{DL}_{50}$ ser menor que $1000 \mu \mathrm{g} / \mathrm{mL}$, dosagem letal máxima para uma substância ser considerada ativa, além de uma correlação estatística entre $\mathrm{a} \mathrm{DL}_{50}$ e a atividade antitumoral (McLaughlin, 1993). Banskota et al. (1998) demonstraram que o extrato metanólico de uma amostra de própolis possui uma citotoxicidade considerável contra células de tumor de cólon, devido ao seu conteúdo de compostos fenólicos. Testes in vitro têm demonstrado atividade antitumoral da própolis tanto citostática quanto citotóxica. 
Tabela 1. Compostos voláteis identificados em amostras de própolis vermelha de Pernambuco (\% do íon corrente total).

\begin{tabular}{|c|c|c|c|c|c|}
\hline \multirow{2}{*}{ Composto } & \multirow{2}{*}{$\mathrm{IK}^{*}$} & \multirow{2}{*}{$\mathrm{IK}^{* *}$} & Fev & Jun & Out \\
\hline & & & \multicolumn{3}{|c|}{ \% área } \\
\hline Hidrocarbonetos monoterpênicos & & & 4,11 & 5,26 & 0,90 \\
\hline$p$-cimeno & 1026 & 1026 & 0,60 & 0,52 & -- \\
\hline limoneno & 1031 & 1031 & 3,51 & 4,74 & 0,90 \\
\hline Monoterpenos oxigenados & & & 1,52 & 3,17 & 1,22 \\
\hline 1,8 -cineol & -- & 1034 & -- & 0,39 & -- \\
\hline linalool & 1098 & 1099 & 1,52 & 2,78 & 1,22 \\
\hline Hidrocarbonetos sesquiterpênicos & & & 32,00 & 17,08 & 20,96 \\
\hline$\alpha$-cubebeno & 1351 & 1355 & 1,17 & 0,33 & 2,49 \\
\hline$\alpha$-copaeno & 1376 & 1382 & 14,70 & 5,97 & 7,27 \\
\hline$\beta$-gurjuneno & 1409 & 1417 & -- & -- & 0,34 \\
\hline$\beta$-cariofileno & 1418 & 1420 & 0,85 & 3,45 & 1,48 \\
\hline$\alpha$-bergamoteno & 1436 & 1440 & 0,51 & 0,88 & 0,49 \\
\hline farneseno & -- & 1420 & 7,54 & 1,82 & 1,16 \\
\hline trans- $\beta$-farneseno & -- & 1458 & 0,58 & -- & 1,02 \\
\hline D-germacreno & 1480 & 1482 & 0,65 & - & -- \\
\hline$\alpha$-selineno & 1494 & 1503 & -- & 0,41 & -- \\
\hline isocarofileno & -- & 1502 & -- & -- & 0,33 \\
\hline$\beta$-bisaboleno & 1509 & 1513 & 6,00 & 2,39 & 5,29 \\
\hline$\delta$-cadineno & -- & 1530 & -- & -- & 1,09 \\
\hline cadineno & 1538 & 1530 & -- & 1,83 & -- \\
\hline Sesquiterpenos oxigenados & & & -- & -- & 0,32 \\
\hline$\delta$-cadinol & -- & 1500 & -- & -- & 0,32 \\
\hline Hidrocarbonetos aromaticos & & & -- & -- & 0,48 \\
\hline naftaleno & -- & 1186 & -- & -- & 0,48 \\
\hline Álcoois, aldeidos e cetonas & & & 20,48 & 6,00 & 9,09 \\
\hline 4-hidroxi-4-metil -heptan-2-ona & -- & 851 & 14,42 & -- & 0,41 \\
\hline 6-metil-5-hepten-2-ona & 985 & 985 & 1,00 & 1,01 & -- \\
\hline octanal & 1001 & 1001 & 0,50 & 0,58 & 0,78 \\
\hline nonanal & 1102 & 1103 & 1,52 & 1,90 & 2,46 \\
\hline$n$-decanal & 1204 & 1205 & 1,14 & 1,31 & 4,49 \\
\hline anisaldeido & 1252 & 1255 & 1,20 & 1,20 & 0,95 \\
\hline n-dodecanal & 1417 & 1409 & 0,70 & -- & -- \\
\hline Hidrocarbonetos alifáticos & & & -- & 1,84 & -- \\
\hline tetradecano & -- & 1399 & -- & 0,55 & -- \\
\hline pentadecano & 1500 & 1499 & -- & 0,85 & -- \\
\hline hexadecano & 1600 & 1598 & -- & 0,44 & -- \\
\hline Fenil propanoides & & & 37,10 & 65,92 & 66,32 \\
\hline estragol & -- & 1200 & 3,62 & 6,16 & 3,61 \\
\hline trans-anetol & 1283 & 1288 & 25,58 & 48,05 & 52,58 \\
\hline metil-cis isoeugenol & 1402 & 1404 & 5,61 & 8,18 & 7,38 \\
\hline trans-metil isoeugenol & 1447 & 1497 & 0,83 & 1,35 & 1,16 \\
\hline elemicin & 1554 & 1555 & 1,46 & 2,18 & 1,59 \\
\hline Não identificados & & & 4,79 & 0,73 & 1,19 \\
\hline \multicolumn{6}{|c|}{$\mathrm{IK}^{*}$ (Índice de Kovats calculado) IK** (Índice de Kovats da literatura). } \\
\hline & & & & & $\begin{array}{l}\text { Rev. Bras. Farmacogn. } \\
\text { Braz. J. Pharmacogn. } \\
\text { 19(2B): Abr./Jun. } 2009\end{array}$ \\
\hline
\end{tabular}




\section{CONCLUSÃO}

A composição química dos compostos voláteis da própolis vermelha do Estado de Pernambuco mostrou-se variada, destacando-se como constituintes majoritários o trans-anetol, $\alpha$-copaeno e o metil cis-isoeugenol. A própolis apresenta como constituintes majoritários os compostos fenólicos, do tipo flavónoides, antraquinonas e fenóis. Estas substâncias na própolis são representadas pelas agliconas de flavonóides, ácidos fenólicos e seus ésteres, os quais são responsáveis pela grande variedade das propriedades biológicas (Banskota et al., 1998; Burdock, 1998; Koo \& Park, 1997; Banskota et al., 2000).

O bioensaio de letalidade em Artemia salina sugere uma promissora atividade antitumoral para esta própolis a partir de seu conteúdo em compostos fenólicos confirmado. Dessa maneira, a expansão da indústria de própolis vermelha só será possível, de forma segura, com a padronização da matéria-prima e dos produtos acabados, levando-se em conta a diversidade da vegetação regional, a atividade de coleta das diferentes variedades de abelhas e com o estabelecimento da eficácia e segurança dos produtos obtidos.

\section{AGRADECIMENTOS}

A CAPES a ao CNPq pelo apoio financeiro e a Mel Brasil pelas amostras de própolis.

\section{REFERÊNCIAS}

Adams RP 1995. Identification of essential oil components by gas chromatography/mass spectroscopy, Allured, Carol Stream, IL, USA.

Aga H, Shibuya T, Sugimoto T, Kurimoto M, Nakagina S 1994. Isolation and identification of antimicrobial compounds in Brazilian propolis. Biosci Biotechnol Biochem 58: 945-946.

Bankova V, Christov R, Kujumgiev A, Marcucci M C, Popov S 1995. Chemical composition and antibacterial activity of Brazilian propolis. Z Naturforsch 50c: 167-172.

Bankova V, Boudourova-Krasteva G, Popov S, Sforcin JM, Funari SRC 1998. Seasonal variations of the chemical composition of Brazilian propolis. Apidologie 29: 361-367.

Bankova V, Castro S, Marcucci MC 2000. Propolis: recent advances in chemistry and plant origin. Apidologie 31: 3-15.

Bankova V, Castro S, Marcucci MC 2000. Propolis: recent advances in chemistry and plant origin. Apidologie 31: 3-15. (repetido acima)

Banskota AH, Tezuka Y, Prasain JK, Matsushige K, Saiki I, Kadota S 1998. Chemical constituents of Brazilian propolis and their citotoxic activities. J Nat Prod 29: 896-900.

Banskota AH, Tezuka Y, Adnyana IK, Midorikawa K, Matsushige K, Message D, Huertas AAG, Kadota S 2000. Cytotoxic, hepatoprotective and free radical scavenging effects of propolis from Brazil, Peru, the Netherlands and China. $J$ Ethnopharmacol 72: 239-246.
Bianchini L, Bedendo IP 1998. Efeito antibiótico da própolis sobre bactérias fitopatogênicas. Sci Agric Piracicaba 55: 149-152.

Burdock GA 1998. Review of the biological properties and toxicity of bee propolis (propolis). Food Chem Toxicol 36: 347-363.

Chang R, Piló-Veloso D, Morais SAL, Nascimento EA 2008. Analysis of a Brazilian green propolis from Baccharis dracunculifolia by HPLC-APCI-MS and GC-MS. Rev Bras Farmacogn 18: 549-556.

Costa, AF 2002. Farmacognosia. 6a ed. Lisboa: Fundação Calouste Gulbenkian.

Dewick, PM 2003. Medicinal Natural Products. Chichester: John Wiley \& Sons.

Dutra RP, Nogueira AMC, Marques RRO, Costa MCP, Ribeiro MNS 2008. Avaliação farmacognóstica de geoprópolis de Melipona fasciculata Smith da Baixada maranhense, Brasil. Rev Bras Farmacogn 18: 557-562.

Harbone JB 1984. Phytochemical methods. 3.ed. London: Chapman \& Hall.

Koo MH, Park YK 1997. Investigation of flavonoid aglycones in propolis collected by two different varieties of bees in the same region. Biosci Biotechnol Biochem 61: 367-369.

Longhini R, Raksa SM, Oliveira ACP, Svidzinski TIE, Franco SL 2007. Obtenção de extratos de própolis sob diferentes condições e avaliação de sua atividade antifúngica. Rev Bras Farmacogn 17: 388-395.

Lustosa SR, Galindo AB, Nunes LCC, Randau KP, Rolim Neto PJ 2008. Própolis: atualizações sobre a química e a farmacologia. Rev Bras Farmacogn 18: 447-454.

Marcucci MC, Ferreres F, García-Viguera C, Bankova VS, De Castro SL, Dantas AP, Valente PHM, Paulino N 2001. Phenolic compounds from Brazilian propolis with pharmacological activities. J Ethnopharmacol 74: 105-112.

Marcucci MC, Trusheva B, Popova M, Vassya Bankova V, Simova S, Miorin PL, Pasin FR, Tsvetkova I 2006. Bioactive constituents of Brazilian red propolis, Advance Access Publication. eCAM 3: 249-254.

McLaughlin JL, Chang CJ, Smith DL 1993. In Human medicinal agents from plants, Kinghorn AD \& Balandrin MF eds., Symposium Series No. 534, American Chemical Society, Washington, D.C. 112-137.

Meyer BN, Ferrigni NR, Putnam JE, Jacobsen LB, Nichols DE, McLaughlin JL 1982. Brine shrimp: A convenient general bioassay for active plant constituents. Planta Med 45: 31-34.

Moersdorf K 1966. Ciclic terpenes and their choleretic action. Clin Ther 7: 442-443.

Nascimento EA, Chang R, Morais SAL, Piló-Veloso D, Reis DC 2008. Um marcador químico de fácil detecção para a própolis de Alecrim-do-Campo (Baccharis dracunculifolia). Rev Bras Farmacogn 18: 379-386.

Nirmala Devi TD, Armugasamy S, Vijayalatha K 2005. Herbs for Good Health. Publications of Centre for Indian Knowledge Systems, Kottupuram, India.

Park YK, Koo MH, Ikegaki M, Contado JL 1997. Comparison of the flavonoid aglycone contents of Apis mellifera propolis from various regions of Brazil. Braz Arch Biol Technol 40: 97-106.

Park YK, Abreu JAS, Ikegabi M, Cury JA, Rosalen PL 1998. Antimicrobial activity of propolis on oral microorganisms. Curr Microbiol 36: 24-29.

Park KY, Alencar SM, Aguiar CL 2002. Botanical origin and 
chemical composition of Brazilian propolis. J Agric Food Chem 50: 2502-2506.

Pereira AS, Seixas FRMS, Neto FRA 2002. Propolis: 100 anos de pesquisa e suas perspectivas futuras. Quim Nova 25: 321-326.

Petri G, Lemberkovics E, Foldvari M 1988. Examination of differences between propolis (bee glue) produced from different floral environments. In Flavours and Fragrances: a world perspective (eds. Lawrence BM, Mookherjee BD, Willis BJ). Elsevier Sci. PubI., Amsterdam, p.439-446.

Pietta PG, Gardana C, Pietta AM 2002. Analytical methods for quality control of propolis. Fitoterapia 73 (Suppl. 1): S720.

Salatino A, Teixeira EW, Negri G, Message D 2005. Origin and chemical variation of Brazilian propolis. eCAM 2: 3338.

Sena Filho JG, Melo JGS, Saraiva AM, Gonçalves AM, Psiottano MNC, Xavier HS 2006. Antimicrobial activity and phytochemical profile from the roots of Lippia alba (Mill.) N.E. Brown. Rev Bras Farmacogn 16: 506-509.

Shukla HS, Tripathi SC 1987. Antifungal substance in the essential oil of anise (Pimpinella anisum L.). Agric Biol Chem 51: 1991-1993.

Simões CC, Araújo DB, Araújo RPC 2008. Estudo in vitro e ex vivo da ação de diferentes concentrações de extratos de própolis frente aos microrganismos presentes na saliva de humanos. Rev Bras Farmacogn 18: 84-89.

Sousa JPB, Furtado NAJC, Jorge R, Soares AEE, Bastos JK 2007. Perfis físico-químico e cromatográfico de amostras de própolis produzidas nas microrregiões de Franca (SP) e Passos (MG), Brasil. Rev Bras Farmacogn 17: 85-93.

Wagner H, Bladt S 1996. Plant drug analysis - A thin layer chromatography atlas; Springer: Munich, 2.ed. 